\title{
Original OAsticle
}

\section{Insulin-Chitosan Polyelectrolyte Nanocomplexes: Preparation, Characterization and Stabilization of Insulin}

\author{
Amani Elsayed ${ }^{1}$, Mayyas Al-Remawi ${ }^{2}$, Asim Farouk ${ }^{3}$, Adnan Badwan ${ }^{4}$
}

\begin{abstract}
Objectives: To formulate chitosan nanoparticles with specific combinations of molecular weight and degree of deacetylation (DDA) that could be developed into an oral insulin delivery system.

Methods: This study was conducted at Jordanian Pharmaceutical Manufacturing Company (JPM), Jordan in the period 2006-2009.

Nanoparticles were prepared by polyelectrolyte complexation method (PEC). The physicochemical characteristics of the nanoparticles were evaluated. The role of nanoparticles in stabilization of insulin at high temperature and protecting insulin from pancreatic degradation was investigated.

Results: The PEC formation process is influenced by a variety of parameters, including the system $\mathrm{pH}$, chitosan molecular weight and DDA. The most important factor appears to be the system $\mathrm{pH}$. All insulin-chitosan complexes displayed positive zeta potential. PECs protect insulin from pancreatin and the protective ability affected by DDA of chitosan. The results of insulin stability indicate that insulin-chitosan PEC protects insulin from degradation for at least $24 \mathrm{~h}$.

Conclusions: Molecular parameters of chitosan nanoparticles play an important role in stabilization of insulin in the GIT. So we can modulate relative parameters to develop an oral insulin delivery system.
\end{abstract}

Key Words: Insulin, Chitosan, Polyelectrolyte complexes, Nanoparticles.

$\mathrm{R}$

ecently, increasingly been investigated as carriers for hydrophilic macromolecular drugs such as peptides, proteins, vaccine and DNA to improve stability and permit administration through non-parenteral routes ${ }^{1-3}$.

Many different hydrophilic nanoparticles have been developed as drug carriers to take advantage of the hydrophilic properties of proteins and peptides. Chitosan a hydrophilic polysaccharide is characterized for its biodegradable, biocompatible, permeation enhancing effect, and mucoadhesive properties providing several pharmaceutical applications $^{4-6}$.

1.Assistant Prof., Faculty of Pharmacy, University of Gezira, Wad Medani, Sudan.

2. Assistant Prof., Suwagh for drug delivery systems, subsidiary of the Jordanian Pharmaceutical Manufacturing Co., Plc., Naor, Jordan

3. Prof., Faculty of Pharmacy, Omdurman Islamic

University,Omdurman, Sudan

4. Dr.General Manager of the Jordanian

Pharmaceutical Manufacturing Company (JPM)
Different methods have been used to prepare chitosan nanoparticles. These include emulsion cross-linking, coacervation/precipitation, spray drying, emulsion coalescence method, reverse micellar method, and ionic gelation ${ }^{7}$.

Previously described insulin-loaded chitosan nanoparticles were prepared by ionotropic gelation of chitosan with tripolyphosphate anions ${ }^{8,}{ }^{9}$. Recently, polyelectrolyte complexation method was utilized to prepare insulin- trimethyl chitosan nanoparticles for intranasal delivery of insulin ${ }^{10}$.

With regard to chitosan-based PEC, nearly all insulin-loaded PEC have been prepared using high molecular weight chitosans or chitosan derivatives. Chitosan is a polycation with an apparent pka 6; hence, in neutral and basic environments prevailing in the intestine, the chitosan molecules lose their charge and precipitated $^{11}$ However, the solubility of chitosan increased with decreasing molecular weight. In addition, Low molecular weight 
chitosans (LMWCs) with high degree of deacetylation possess high reactivity due to a greater number of amino groups available for interactions with the anionic actives ${ }^{12}$. Moreover, their intestinal absorption is known to be significantly better than the high molecular weight candidates and showed negligible cytotoxic effect on the Caco-2 cells ${ }^{13}$.

Hence the main aim of this work was to formulate LMWC nanoparticles with specific combinations of molecular weight and degree of deacetylation that could be developed into an oral insulin delivery system. Initially, insulinchitosan PECs were prepared. The physicochemical characteristics of the nanoparticles were evaluated. In addition, the influence of both molecular weight (M.wt), in the low range, and DDA of chitosan on nanoparticle properties and insulin stability were studied simultaneously. Furthermore, the role of chitosan in protecting insulin from pancreatic degradation was also investigated.

\section{Materials and Methods}

\section{Materials}

Two chitosan grades were used in the study: the commercial low molecular weight chitosan (LMWC) molecular weight $<5 \mathrm{KDa}$ and degree of deacetylation DDA of $81 \%$, Shanghai Nicechem Co, China, b) molecular weight $<10 \mathrm{KDa}$ and DDA of $80 \%$, Shanghai Nicechem Co, China, and c) molecular weight $<50 \mathrm{KDa}$ and DDA of $82 \%$, Shanghai Nicechem Co, China and high molecular weight chitosan (HMWC) MWT $>250 \mathrm{KDa}$ and DDA 95\%, Xiamen Xing, China. Pancreatin (activity equivalent to $4 \mathrm{x}$ USP, lot No.044 k1660) was purchased from SigmaAldrrich, Germany.

\section{Methods}

Preparation and characterization of low molecular weight chitosan (LMWC)

Depolymerization of high molecular weight chitosan.

Ten grams of the high molecular weight chitosan were dissolved in $1 \mathrm{~L}$ of $2 \mathrm{M} \mathrm{HCl}$. The solution was heated for $3.5 \mathrm{~h}$ under reflux. After cooling, $3 \mathrm{~L}$ of ethanol $(96 \%)$ was added and the precipitate was washed thoroughly with ethanol and freeze dried for
$24 \mathrm{~h}$. The resultant chitosan powder was identified by Fourier transform infrared (FTIR) spectroscopy.

Determination of molecular weight.

The viscosity of chitosan hydrochloride dissolved in water was measured using a viscometer (Vibro viscometer, SV-10, Japan), and the viscosity average molecular weight was deduced using the Mark-Houwink's equation, $(\eta)=K\left(M_{v}\right)^{a}$ where $(\eta)$ is the intrinsic viscosity, $M$ is the viscosity average molecular weight, and $\mathrm{k}$ and a values were 0.00058 and 0.69 based on a previous study ${ }^{14}$. Determination of degree of deacetylation.

The degree of deacetylation (DDA) was determined according to spectroscopic method of the British Pharmacopoeia (B.P) 2007.

\section{Preparation and characterization of insulin-chitosan polyelectrolyte complex (PEC)}

Insulin-chitosan complex preparation. Chitosan with volume of $0.5 \mathrm{~g}$ was placed in a glass vial, dissolved in $10 \mathrm{~mL}$ deionized water and its $\mathrm{pH}$ was adjusted to 5.5 using about $4 \mathrm{~mL}$ of $0.2 \mathrm{M} \mathrm{NaOH}$, and the final volume was completed to $20 \mathrm{~mL}$ using deionized water. In another vial, $100 \mathrm{mg}$ of rh-insulin powder was dissolved in $1 \mathrm{~mL}$ of $0.1 \mathrm{M} \mathrm{HCl}$, followed by the addition of $3 \mathrm{~mL}$ of $1 \mathrm{M}$ tris (hydroxymethyl)-aminomethane buffer $\mathrm{pH}$ 7. Equal volumes of the chitosan and insulin were stirred gently using magnetic stirrer for 15 min at $4{ }^{\circ} \mathrm{C}$.

.Insulin-chitosan association efficiency $(A E)$.

To determine the association efficiency of insulin with chitosan, triplicate batches of insulin-chitosan polyelectrolyte complex were centrifuged at $15000 \mathrm{rpm}$ for $30 \mathrm{~min}$ at $15{ }^{\circ} \mathrm{C}$ and the insulin content in the supernatant was assayed by reversed phase high pressure liquid chromatography (RPHPLC) as mentioned earlier ${ }^{15}$. The association efficiency was calculated as described elsewhere ${ }^{16}$ using the following equation:

Association efficiency $=$

Total amount of insulin-Free insulin X 100\%

Total amount of insulin 
The factors that might affect the AE, were studied, namely, final $\mathrm{pH}$ of the complex $(6.5$ or 6.8), molecular weight of chitosan $(2.3,3$, 13 or $30 \mathrm{KDa})$, DDA of chitosan $(80$ or $100 \%)$, and chitosan: insulin ratio $(1: 1,2: 1$ or $3: 1)$.

Determination of particle size and zeta potential of insulin-chitosan complex

The particle size distribution of nanoparticles was carried out with Zetasizer Nano-ZS (Malvern Instruments, UK) at $25{ }^{\circ} \mathrm{C}$. Scattering light was detected at $173^{\circ}$ angle. For data analysis, the viscosity $(0.88 \mathrm{mPa} . \mathrm{s})$ and the refractive index (1.33) of pure water at $25^{\circ} \mathrm{C}$ were used.

The zeta potential measurements were acquired using Zetasizer Nano ZS at $25^{\circ} \mathrm{C}$. Samples of free chitosan and insulin-chitosan PEC were measured in folded capillary cells integrated with gold electrodes. Three measurements were conducted, and the number of runs in each measurement was automatically determined by the software. Smoluchowski approximation was used and the results were expressed as mean $\pm \mathrm{SD}$. The viscosities of samples were measured by Sine wave Vibro viscometer (SV-10, A\&D Company, Japan) at $25^{\circ} \mathrm{C} \pm 0.01$ in triplicate.

\section{Effect of temperature on insulin stability}

Determination of melting temperature of insulin.

Melting temperature measurements were carried out with Zetasizer Nano ZS using a 1 ${ }^{\circ} \mathrm{C}$ incremental temperature ramp and a $3 \mathrm{~min}$ equilibrium time at each melting temperature measurement. Insulin solutions in tris buffer with the concentration of $1 \mathrm{mg} / \mathrm{mL}$ and $\mathrm{pH} 7$ \pm 0.1 were prepared and their melting temperature was determined. The marked point where both the size and the intensity start to increase significantly is called melting temperature $(\mathrm{Tm})$.

Effect of temperature on the stability of insulin-chitosan PEC.

To evaluate the effect of LMWC on the stability of insulin, accelerated stability testing was conducted. PEC suspension with volume of $10 \mathrm{~mL}$ was prepared as mentioned above except that the final concentration of both insulin and chitosan was adjusted to 500 $\mathrm{lg} / \mathrm{mL}$. PEC suspension was incubated in a water bath shaker at $55 \pm 1{ }^{\circ} \mathrm{C}$ with shaking of 100 strokes $/ \mathrm{min}$. At predetermined time points $(0,12,24,48 \mathrm{~h})$, aliquots were withdrawn and diluted with $0.01 \mathrm{M} \mathrm{HCl}$ to dissolve the nanoparticles. The insulin content was determined by RP-HPLC as described earlier. In addition, the stability of a standard insulin solution was assessed under the same testing conditions. All samples were prepared in triplicate.

Enzymatic stability studies with pancreatin In order to investigate the role of chitosan in protecting insulin from pancreatic degradation, insulin-chitosan complexes were prepared from chitosans of different molecular weight and degree of deacetylation. $1 \mathrm{ml}$ of the complex or free insulin was incubated with $4 \mathrm{ml}$ of SIF with pancreatin according to USP 2007 at $37{ }^{\circ} \mathrm{C}$ while shaking at 100 strokes/min. Aliquot volume of $100 \mu \mathrm{l}$ was taken after $1 \mathrm{hr}$ and samples were diluted with $0.01 \mathrm{M} \mathrm{HCl}$ to terminate the pancreatic action and analyzed by RP-HPLC. The average and standard deviation of three replicates were calculated.

\section{Results and discussion}

Depolymerization, molecular weight and degree of deacetylation determination

Depolymerization of high molecular weight chitosan by hydrochloric acid was carried out according to previously reported methods ${ }^{17}$, 18. This yielded fractions of low molecular weight chitosans. Identification of these fractions was carried out by FTIR which showed similar distinct peaks as appeared in the parent compound. This evidenced that depolymerization process did not change the basic chemical structure.

The degree of deacetylation of depolymerized and commercial chitosans was determined by first derivative spectroscopic (FDUV) method. As shown in Table 1 DDA of depolymerized fractions were around 100\% which indicated that the samples were completely deacetylated. 
Table 1. Degree of deacetylation of chitosans

\begin{tabular}{c|ccc}
$\begin{array}{c}\text { Chitosan M. wt } \\
(\text { KDa })\end{array}$ & Source & $\begin{array}{c}\text { DDA calculated by } \\
\text { FDUV method }\end{array}$ & $\begin{array}{c}\text { DDA calculated by } \\
\text { NMR method }\end{array}$ \\
\hline 3 & Depolymerized & $99.80 \pm 0.05$ & 100 \\
13 & Depolymerized & $99.90 \pm 0.38$ & 100 \\
28 & Depolymerized & $99.00 \pm 0.71$ & 100 \\
2.3 & Commercial & $84.28 \pm 0.42$ & 83.33 \\
8 & Commercial & $83.00 \pm 0.63$ & 82 \\
30 & Commercial & $82.48 \pm 0.31$ & 78.74
\end{tabular}

The high values obtained were in an agreement of a previous report ${ }^{19}$. There is slight increase in the degree of deacetylation of depolymerized LMWC when compared to the native high molecular weight chitosan (DDA 93\%), indicating that hydrochloric acid can hydrolyzed $O$-glycosidic linkages (depolymerization) as well as the $N$-acetyl linkage (de-N-acetylation) though at different rates $^{20}$.

\section{Preparation of insulin-chitosan PEC}

Insulin and chitosan mixture was prepared through the gradual addition of the two solutions containing materials with opposite charges to facilitate the formation of the PEC. However, PEC preparation was previously reported for polymers having ionizable groups and bear opposite charges ${ }^{21}$.

Insulin-chitosan PEC association efficiency

Effect of $p H$ on $A E$.

Chitosan having low molecular weight and high degree of deacetylation is highly active due to a greater number of amino groups available for interactions with the anionic active sites ${ }^{12}$. Chitosan solution $\mathrm{pH}$ was adjusted to 5.5. At this $\mathrm{pH}$ about $90 \%$ of the amine groups are protonated as it has an apparent $\mathrm{pKa}$ of $\sim 6.5^{11}$. Such protonation leads to chain repulsion and more extended conformations $^{22}$. This exposes the amine groups to the negatively charged insulin leading to their interaction. Insulin has an apparent isoelectric point (pI) of 6.4 and a charge of -2 at a final $\mathrm{pH}$ of $\sim 6.8^{23}$. The influence of the final $\mathrm{pH}$ on association efficiency was evaluated as presented in Table 2.
Table 2: Effect of $\mathrm{pH}$ and insulin to chitosan weight ratio on $\mathrm{AE}$ (average \pm standard deviation), $\mathrm{n}=3$

\begin{tabular}{c|c}
$\mathrm{pH}$ & $\mathrm{AE} \pm \mathrm{SD}$ \\
\hline 6.5 & $78.81 \pm 0.68$ \\
6.8 & $30.38 \pm 3.71$ \\
\hline Ratio (w/w) & $\mathrm{AE} \pm \mathrm{SD}$ \\
\hline $1: 1$ & $78.81 \pm 0.68$ \\
$1: 2$ & $73.38 \pm 3.42$ \\
& $77.11 \pm 3.94$ \\
\end{tabular}

*Chitosan $13 \mathrm{KDa}, \mathrm{DDA}=99.9$

High AE was obtained at pH 6.5 (about 80\%). It was reported that the formation of PEC between the two oppositely charged polymers can only occur at $\mathrm{pH}$ values in the vicinity of the pKa interval of the two polymers ${ }^{21}$. The $\mathrm{AE}$ fell sharply when the final $\mathrm{pH}$ of the complex was adjusted to 6.8 (about 30\%). This finding is in agreement with studies of Ma et $\mathrm{al}^{24}$ who recorded a sharp change in $\mathrm{AE}$ with small $\mathrm{pH}$ changes. It seems that chitosan becomes increasingly globular as the $\mathrm{pH}$ increases, and starts to precipitate ${ }^{22}$.

Effect of molecular weight and DDA

It is expected that the spread length of chitosan chain in solution may vary in 
correlation with the molecular weight, which may affect protein interaction and encapsulation. Longer chains may offer more reactive sites for the formation of hydrogen bonds with protein molecules, especially if a protein maintains a compact structure and not fully extended at the solution conditions ${ }^{25}$. On the other hand, It is expected that shorter chains have greater flexibility and therefore more preferable for interaction ${ }^{12}$.

The effect of chitosan molecular weight on the $\mathrm{AE}$ was evaluated (Figure 1). Low association efficiency for the shorter chain chitosans (2.3 and $3 \mathrm{KDa}$ ) was observed. Optimum polymer /insulin (+/-) charge ratio was essential for PEC formation ${ }^{26}$.

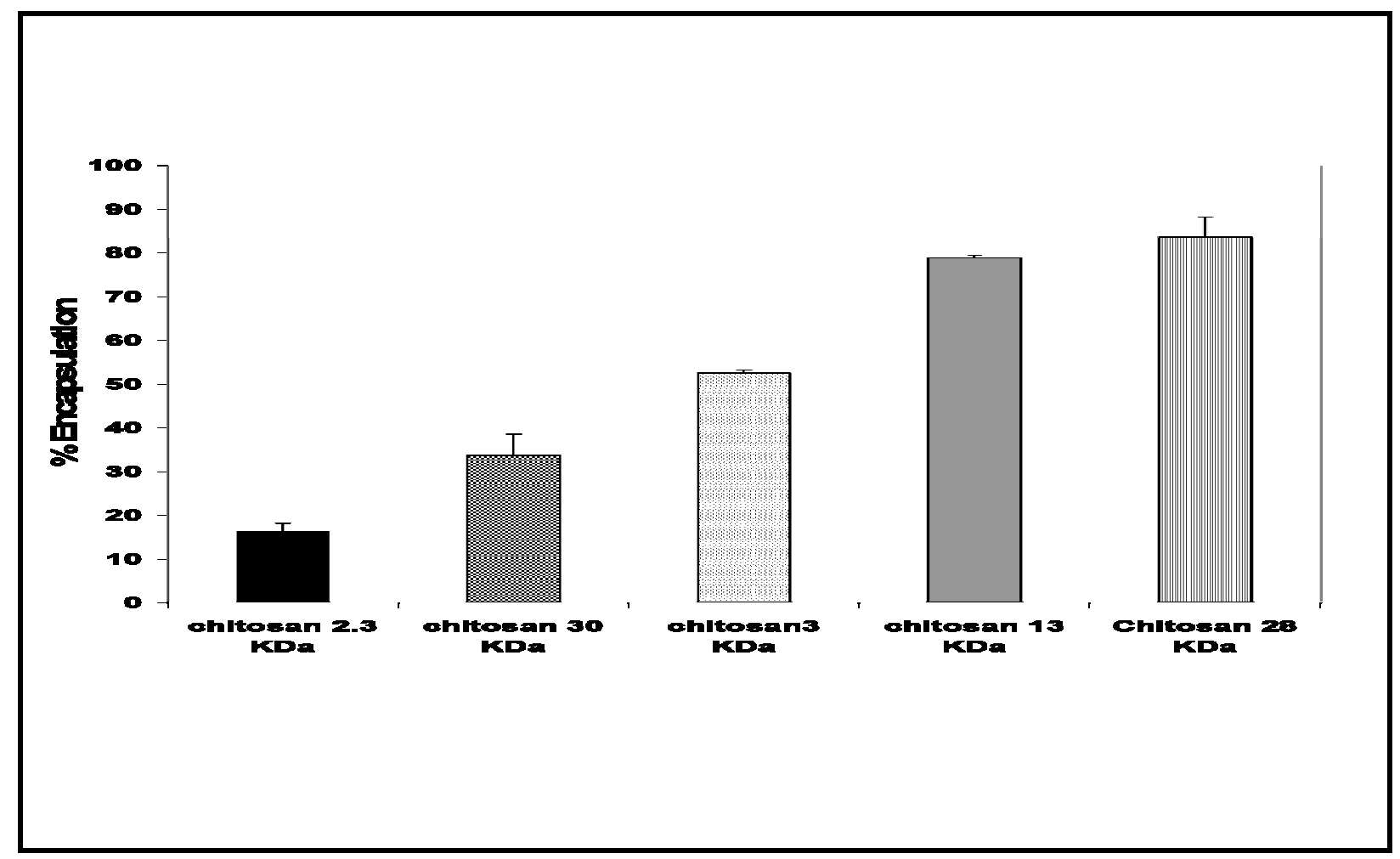

Figure 1. Effect of M.wt and DDA of chitosan on the association efficiency of insulin

The same weight ratio (1 to 1 chitosan to insulin) was used in this comparative study, the decrease in AE for chitosans (2.3 and 3 $\mathrm{KD})$ could be attributed to lower charge densities on shorter chain chitosans compared to the other chitosans. For example zeta potential for chitosan 2.3 and $30 \mathrm{KDa}$ was +13.71 and +27.87 respectively.

AE was also affected by the DDA of chitosan, as the DDA increases the encapsulation increases as well (Figure1). This is to be expected as higher DDA may offer more reactive sites for electrostatic interaction with insulin

Effect of chitosan to insulin ratio on $A E$.

The AE was not affected when chitosan to insulin ratio was increased from 1: 1 to $3: 1$ as shown in Table 2. This finding is inconsistent with some previous studies ${ }^{27,}{ }^{28}$. This contradiction may be attributed to the differences in the chitosan molecular weight used and their purity. Chitosan /insulin charge ratio (+/_) was calculated according to the charge and $\mathrm{pKa}$ of the components at $\mathrm{pH} 6.5$. It was found 9:1 and 27:1 for chitosan/insulin, $1: 1$ and $3: 1 \mathrm{w} / \mathrm{w}$ ratio, respectively. The positive charge of chitosan was present in molar excess, and may partially explain the constant $\mathrm{AE}$ at different chitosan to insulin ratios.

However, it is not only the charge but chitosan conformation and viscosity were also known to be affected upon the increase in chitosan concentration in solution state. All of these factors could influence the interaction of chitosan and insulin and, hence, affecting the 
AE. It was reported that chitosan at high concentration adopts a random coil conformation, and the proportions of the charged segments are shielded due to strong intermolecular interactions ${ }^{29}$. In addition, the viscosity of chitosan increases with the increase in chitosan concentration in solution $^{22}$. This may hamper the kinetics and led to a limited binding with insulin.

\section{Particle size and Zeta potential.}

An increase in the molecular weight of the chitosan polymer led to an increase in the dispersed phase particle size as shown in
Table 3. This finding is in agreement with other published studies ${ }^{10}$.

As expected the zeta potential of chitosan is positive due to presence of amino groups. All chitosan-insulin complexes displayed a lower positive zeta potential in comparison with the free chitosan. The ratio of negative charge (insulin) to the positive charge of the chitosan remains in the favor of the positive charge of the polymer as only a fraction is neutralized by binding insulin. This indicated that partial neutralization of chitosan charge took place. These results agree well with the results reported by Sadeghi et $\mathrm{al}^{16}$.

Table 3. Particle size and Zeta potential of free chitosan and chitosan-insulin PEC prepared from different chitosan molecular weights and DDA.

Chitosan

M.wt (KDa)

$\begin{array}{lll}2.3 & 81 \pm 0.42 & 63 \pm 4.0 \\ 30 & 82 \pm 0.31 & 141 \pm 15.6 \\ 3 & 100.8 \pm 0.04 & 79 \pm 2.5 \\ 13 & 99.9 \pm 0.38 & 111 \pm 6.9 \\ 30 & 100 \pm 0.71 & 205 \pm 2.6\end{array}$

\section{Insulin stability}

Insulin is a labile protein similar to other proteins. They are affected by formulation components, process and storage temperature $^{30}$. It is well established that all proteins unfold above their melting temperature $\mathrm{Tm}^{30}$. In order to assess insulin stability the temperature used for testing
Zeta Potential $(\mathrm{mv}) \pm \mathrm{SD}$

\begin{tabular}{ll} 
Free Chitosan & Chitosan-Insulin \\
\hline $13.17 \pm 1.41$ & $7.93 \pm 0.48$ \\
$27.87 \pm 0.45$ & $12.30 \pm 1.47$ \\
$27.6 \pm 0.62$ & $7.07 \pm 0.29$ \\
$56.83 \pm 0.31$ & $19.97 \pm 1.65$ \\
$51.7 \pm 2.18$ & $21.77 \pm 1.15$
\end{tabular}

should be far enough from its Tm. Consequently, Tm of insulin should be first measured prior to accelerated stability testing. Melting point of insulin in tris buffer

Different techniques have been used to determine $\mathrm{Tm}$, such as differential scanning calorimetry, infrared spectroscopy and capillary electrophoresis $31 \quad, 32$.

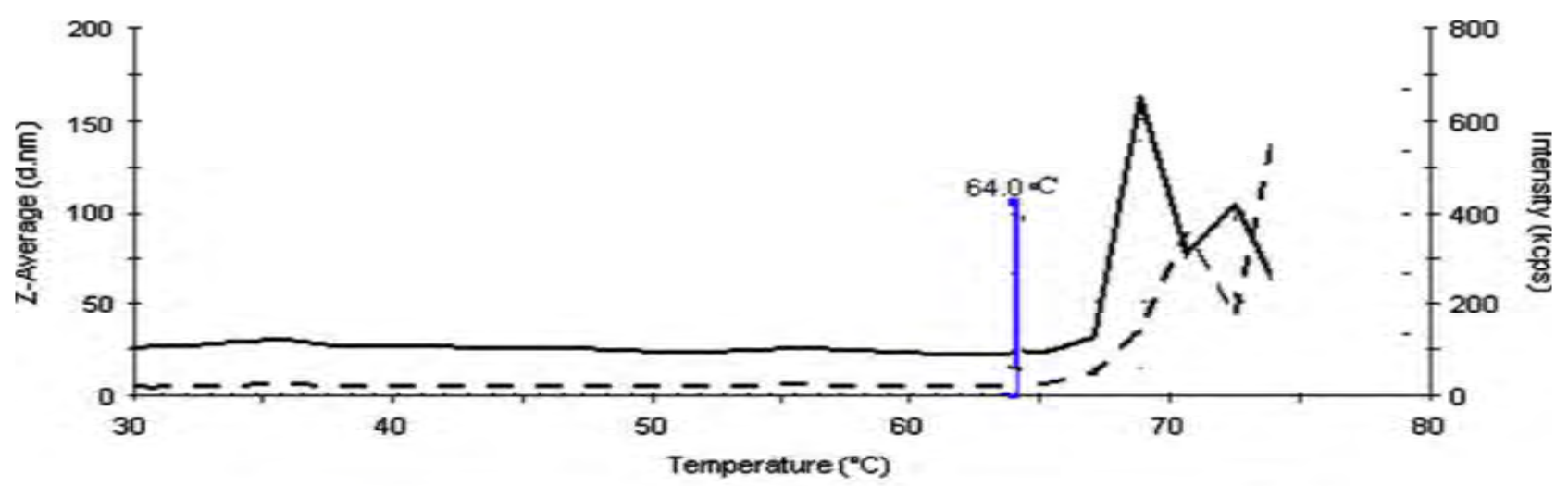
\begin{tabular}{|ll}
$-\ldots-$ & Z-Average (insulin melting point $1 \mathrm{mg}$ per $\mathrm{ml}$ in tris buffer $0.5 \mathrm{M}$ ) \\
\hline & Melting Point (insulin melting point $1 \mathrm{mg}$ per $\mathrm{ml}$ in tris butfer $0.5 \mathrm{M}$ ) \\
Intensity (insulin melting point $1 \mathrm{mg}$ per $\mathrm{ml}$ in tris buffer $0.5 \mathrm{M}$ )
\end{tabular}

Figure 2. Melting temperature of insulin dissolved in $0.5 \mathrm{M}$ tris (hydroxymethyl)-aminomethane buffer. 
Recently, dynamic light scattering technique (DLS) was used, when denaturation occurs, the small

size of the protein is increased to a value consistent with a random coil polymer of the same molecular weight. In the absence of chaotropic (aggregation prohibiting) agents, inter-polymer hydrophobic interactions may quickly lead to non-specific aggregation of the denatured polypeptide chains. The change in size that accompanies the protein denaturation is easily identified using DLS. Figure 2 shows the melting temperature profile for insulin dissolved in tris buffer. At temperatures less than $60{ }^{\circ} \mathrm{C}$, the size and scattering intensity are constant, suggesting a stable tertiary structure. At temperatures higher than $61^{\circ} \mathrm{C}$, both the size and scattering intensity increase exponentially with temperature, indicating the presence of denatured aggregates. The Tm determined by DLS is in agreement with that reported in literature using DSC technique ${ }^{33}$. The average Tm of insulin obtained in this study was $63 \pm$ $1{ }^{\circ} \mathrm{C}$.

Accelerated stability of insulin-chitosan PEC To illustrate the role of LMWC on stabilization of insulin, insulin-chitosan PEC (Chitosan $13 \mathrm{KDa}$ ) was subjected to $55{ }^{\circ} \mathrm{C}$ temperature which is $8^{\circ} \mathrm{C}$ less than the Tm of insulin and was compared with the free insulin solution. It is worth mentioning that other investigators used temperatures $\geq 40{ }^{\circ} \mathrm{C}$ to study the stability of insulin preparations ${ }^{34}$. After $48 \mathrm{~h}$ insulin solution was almost completely degraded as shown in Figure 3.

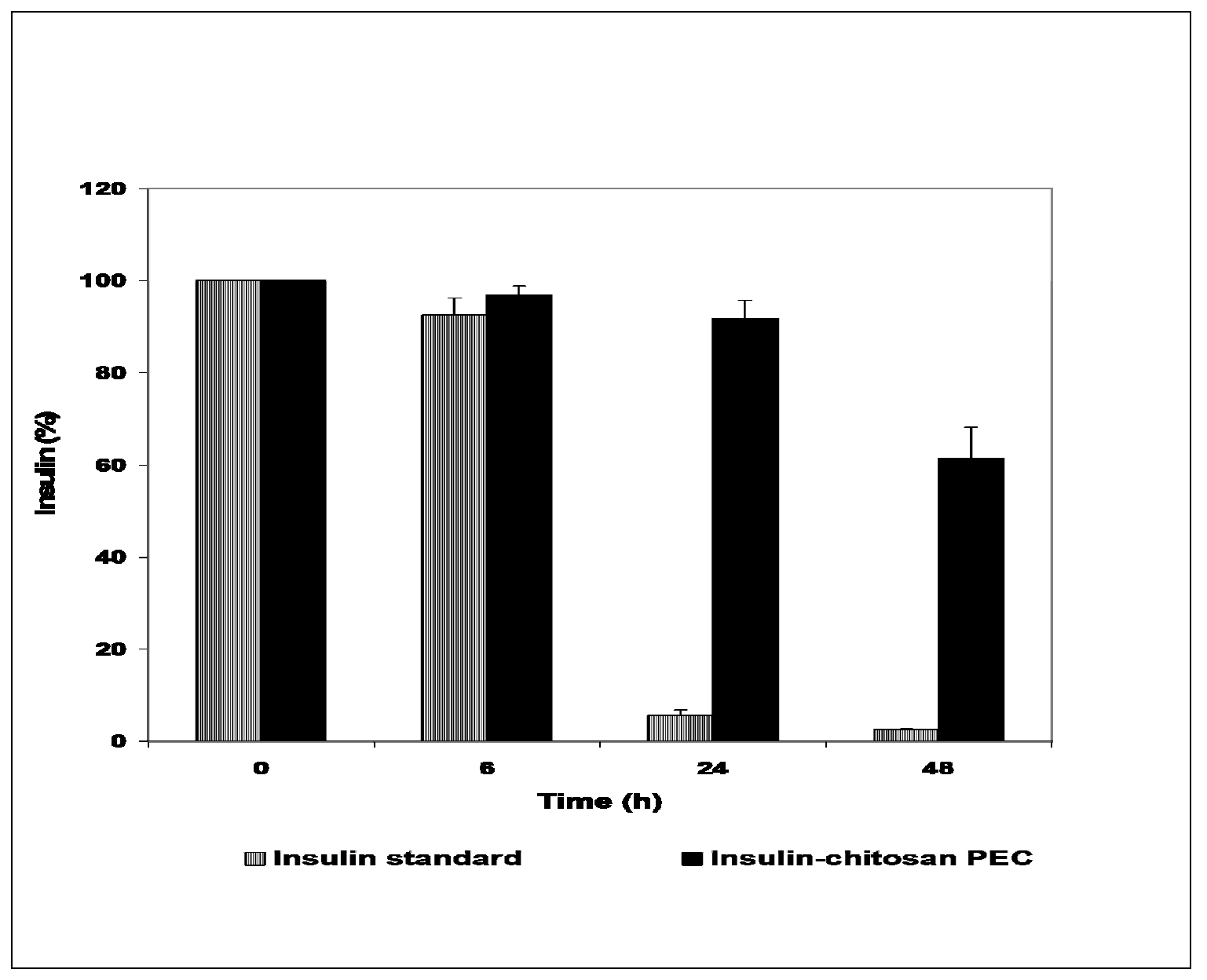

Figure 3. Stability of free insulin and insulin-chitosan PEC placed on a water bath shaker at a temperature of $55^{\circ} \mathrm{C}$ with 100 strokes/min. 
High temperature causes denaturation of insulin by the rapid formation of bovine insulin fibrils ${ }^{30}$. In addition, high temperature accelerates its chemical degradation. It was reported that formation of insulin oligomers and polymers increases at temperature $\geq 25$ ${ }^{\circ} \mathrm{C}^{35}$, while shaking creates hydrophobic air/water interface and, therefore, initiate aggregation $^{36}$. However, insulin-chitosan PEC protects insulin from degradation for at least $24 \mathrm{~h}$. Although high molecular weight chitosan and chitosan derivatives were found to enhance the thermal stability of insulin ${ }^{37}$, the effect of chitosan low molecular weight has yet to be investigated. The protective effect of PEC may be due to the reduction of aggregation and fibrillation of insulin as free insulin solution becomes turbid after $24 \mathrm{~h}$. Other cationic polymers such as poly (ethylene glycol)-b-poly (L-histidine) that forms PEC with insulin reduce aggregation of insulin on agitation ${ }^{38}$. Consequently, one can assume that LMWC reduces insulin aggregations. In addition, Mao et al. suggested that higher temperature causes compaction of insulin nanoparticles ${ }^{10}$. However, the influence of LMWC is still wide open for investigation.

\section{Stability of insulin-chitosan (Ins-Cs) complex in simulated intestinal fluid (SIF)}

The potential role of chitosan in protecting insulin from enzymes present in the small intestine was evaluated. The enzymatic stability of insulin was investigated in the presence of pancreatin. Figure $\mathbf{4}$ depicts the percentage of undegraded insulin after incubation of insulin-chitosan PECs (1 to 1 ratio) prepared from chitosans of different molecular weight and degree of deacetylation with pancreatin for $1 \mathrm{hr}$ and compared to free insulin solution.

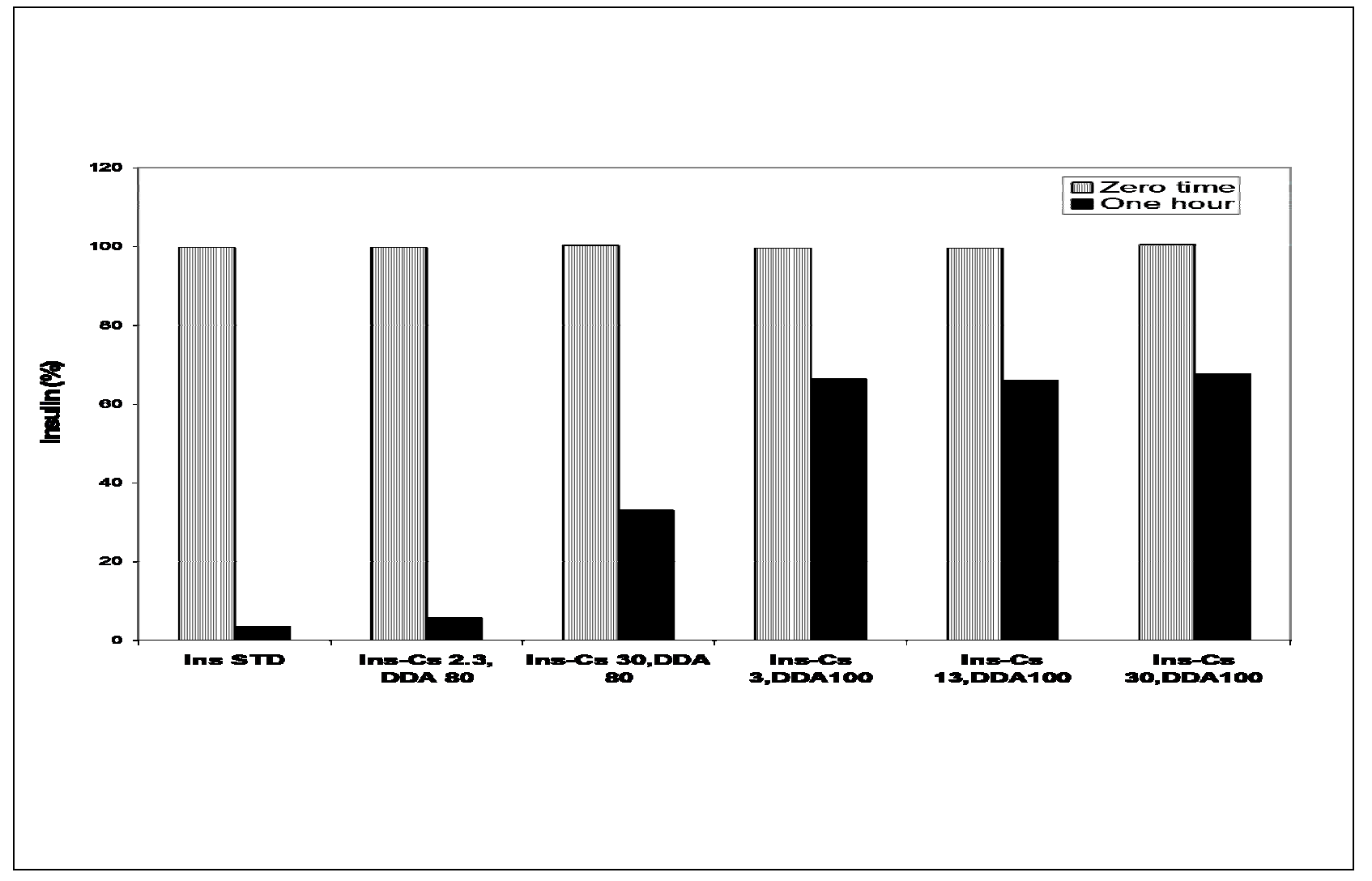

Figure 4. Enzymetic degradation of insulin by pancreatin (USP 2007). Each value represents the mean \pm S.D. $n=3$. 
Partial protection of insulin from pancreatin was observed with chitosans of $100 \%$ DDA $(\mathrm{P}<0.05)$ with relatively similar values (about 60\%) for the M.wt. 3, 13 and $28 \mathrm{KDa}$, whereas, lower protection was manifested for chitosans with DDA $80 \%$. The higher M.wt $30 \mathrm{KDa}$ displayed significant protection compared to free insulin $(\mathrm{P}<0.05)$ in contrast the lower molecular weight $2.3 \mathrm{KDa}$ did not protect insulin from pancreatic digestion under similar conditions $(\mathrm{P}>0.05)$. The significant difference in enzymatic protection of insulin between chitosans with DDA $100 \%$ and DDA $80 \%(\mathrm{P}<0.05)$ could be attributed to the higher percentage of encapsulated insulin in the case of chitosans with DDA $100 \%$.

Another experiment was conducted to evaluate the effect of increasing chitosan to insulin ratio on enzymatic protection. PECs composed of chitosan (13 KDA, DDA, 99\%) to insulin ratios 3 to 1,2 to 1 and 1 to 1 were prepared and compared with free insulin solution as shown in Figure 5 .

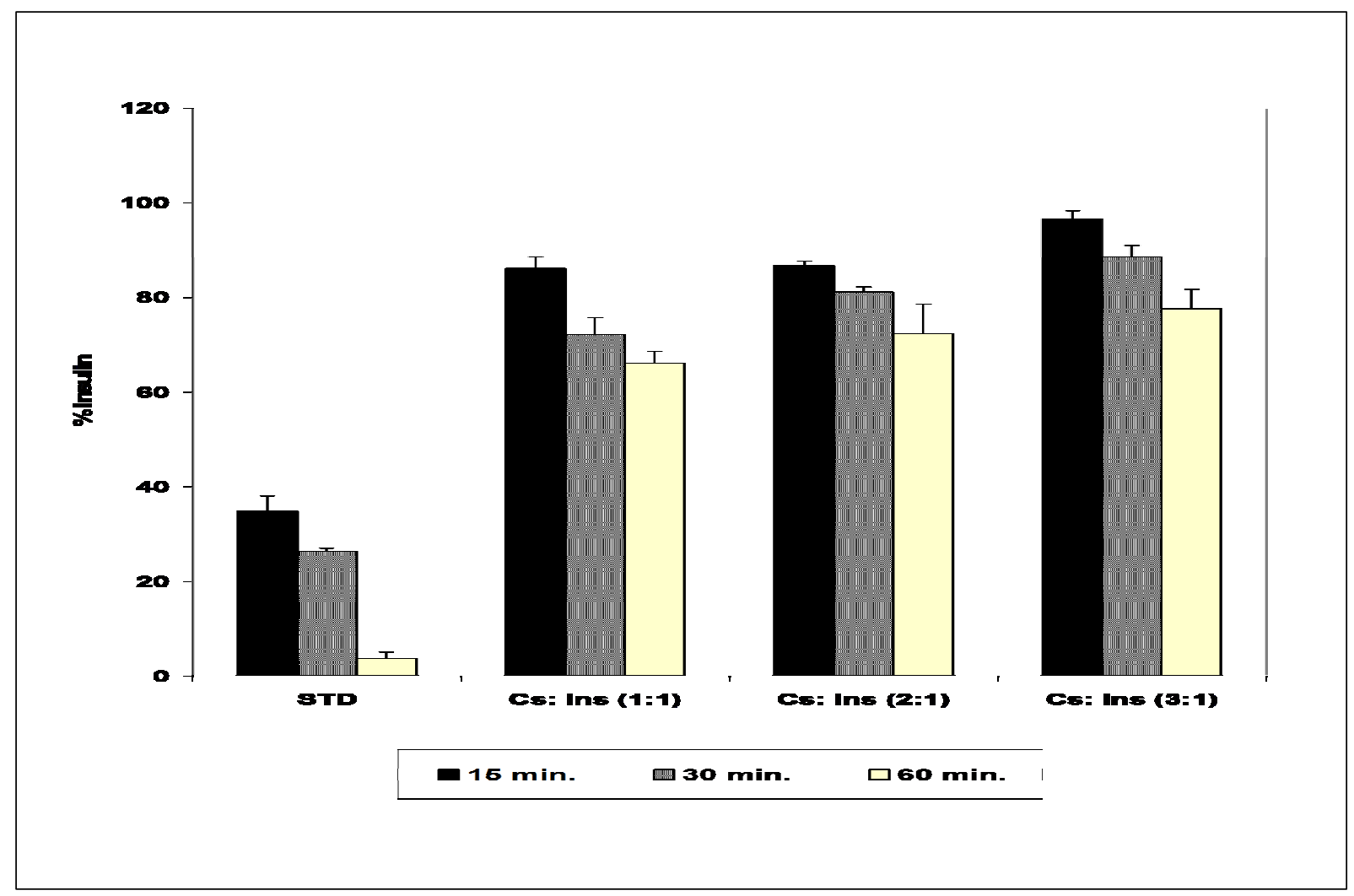

Figure 5. Effect of chitosan:Insulin ratio on pancreatic degradation of insulin

A pronounced enzymatic protective effect of PECs was noted compared to free insulin solution $(\mathrm{P}<0.05)$. The protection increases with increasing chitosan ratio. A significant difference between chitosan-insulin ratios 3 to 1 and 1 to $1(\mathrm{P}<0.05)$ was observed at all time intervals. As discussed earlier there is no significant difference in the encapsulation efficiency between different ratios. The significant difference in enzymatic protection could be due to excess positive charge of the higher ratio leading to repulsion of trypsin as it is also positively charged ${ }^{39}$. Another possible explanation is that phosphate ions of phosphate buffer could bind to the positively polymer and form a gel layer that hinder the penetration of the enzyme. As the ratio of chitosan increases, the thickness of this layer was expected to increase.

\section{Conclusion}

Insulin-chitosan PECs were prepared using LMWT chitosans. Nanoparticles with specific combinations of molecular weight and degree of deacetylation were dispersed in an oily vehicle 
and an oral insulin delivery system was developed by our group. In vivo tests were conducted both on animals and humans ${ }^{40,41}$.

\section{References}

1. Vila A, Sánchez A., Tobío M. et al. Design of biodegradable particles for protein delivery. J. Control. Release 2002; 78: 15-24.

2. Nagamoto T., Hattori Y., Takayama, K. et al. Novel chitosan particles and chitosan-coated emulsions inducing immune response via intranasal vaccine delivery. Pharm. Res.2004; 21: 671-674.

3. Lavertu M, Methot S, Tran-Khanh $\mathrm{N}$ et al. High efficiency gene transfer using chitosan/DNA nanoparticles with specific combinations of molecular weight and degree of deacetylation. Biomaterials 2006; 27: 4815-4824.

4. Muzzarelli R, Baldassarre V, Conti F et al. Biological activity of chitosan: ultrastructural study. Biomaterials 1988; 9: 247-52.

5. Illum L., Farraj N.F Davis, S. Chitosan as a novel nasal delivery system for peptide drugs. Pharm. Res.1994; 11: 1186-1189.

6. Bernkop-Schnurch, A. Chitosan and its derivatives: potential excipients for peroral peptide delivery systems. Int. J. Pharm.2000; 194: 1-13.

7. Agnihotri S., Mallikarjuna N. Aminabhavi, T. Recent advances on chitosan-based micro-and nanoparticles in drug delivery. J. Control. Release 2004; 100: 5-28.

8. Pan Y., Li Y.J., Zhao H.Y. et al. Bioadhesive polysaccharide in protein delivery system: chitosan nanoparticles improve the intestinal absorption of insulin in vivo. Int. J. Pharm.2002; 249: 139-147.

9. Ma Z., Lim, T.M. Lim, L.Y. Pharmacological activity of peroral chitosan-insulin nanoparticles in diabetic rats. Int. J. Pharm.2005; 293: 271-280.

10. Mao S., Bakowsky U., Jintapattanakit A. et al. Selfassembled polyelectrolyte nanocomplexes between chitosan derivatives and insulin. J. Pharm. Sci 2006; 95: 1035-1048.

11. Hejazi R. Amiji, M. Chitosan-based gastrointestinal delivery systems. J. Control. Release 2003; 89: 151165.

12. Sabnis S. Block, L. Chitosan as an enabling excipient for drug delivery systems. 1. Molecular modifications. International journal of Biological Macromolecules 2000; 27: 181-186.

13. Chae S., Jang M., Nah J. Influence of molecular weight on oral absorption of water soluble chitosans. J. Control. Release 2005; 102: 383-394.

14. Kasaai M.R. Calculation of Mark-Houwink-Sakurada .MHS; equation viscometric constants for chitosan in any solvent-temperature system using experimental reported viscometric constants data. Carbohydr. Polym.2007; 68: 477-488.

15. $\mathrm{Xu} \mathrm{X,} \mathrm{Fu} \mathrm{Y,} \mathrm{Hu} \mathrm{H}$, et al. Quantitative determination of insulin entrapment efficiency in triblock copolymeric nanoparticles by high- performance liquid chromatography. J Pharm Biomed Anal. 2006, 41: 266-273.

16. Sadeghi A, Dorkoosh F, M. Avadi, M, et al. Preparation, characterization and antibacterial activities of chitosan, Ntrimethyl chitosan .TMC; and N-diethylmethyl chitosan .DEMC;nanoparticles loaded with insulin using both the ionotropic gelation and polyelectrolyte complexation methods. Int J Pharm. 2008, 355: 299-306.

17. Lee, M, Var F, Shin-ya Y, Kajiuchi, T, et al. Optimum conditions for the precipitation of chitosan oligomers with DP 5-7 in concentrated hydrochloric acid at low temperature. Process Biochem. 1999, 34: 493-500.

18. Varum K, Ottoy, M, Smidsrd, O. Acid hydrolysis of chitosans, Carbohydr Polym. 2001, 46: 89-98.

19. Kumar B. Varadaraj M. Tharanathan, R. Low molecular weight chitosan--preparation with the aid of pepsin, characterization, and its bactericidal activity. Biomacromolecules 2007; 8: 566-572.

20. Varum K., Attoy M. Smidsrod O. Acid hydrolysis of chitosans. Carbohydr. Polym. 2006; 46: 89-98.

21. Berger J., Reist M., Mayer, J. et al. Structure and interactions in chitosan hydrogels formed by complexation or aggregation for biomedical application. Eur. J. Pharm. Biopharm.2004; 57: 35-52.

22. G.A.F. Roberts. Chitin Chemistry, The Mac Millan Press, London, 1992. pp. 1-7.

23. Kaarsholm N.C., Havelund S., Hougaard P. Ionization behavior of native and mutant insulins: $\mathrm{pK}$ perturbation of B13-Glu in aggregated species. Arch Biochem Biophy.1990; 283: 496-502.

24. Ma Z., Yeoh H.H. Lim, L.Y. Formulation $\mathrm{pH}$ modulates the interaction of insulin with chitosan nanoparticles. J. Pharm. Sci.2002; 91: 1396-1404.

25. Gan, Q. Wang, T. Chitosan nanoparticle as protein delivery carrier--systematic examination of fabrication conditions for efficient loading and release. Colloids Surf. B Biointerfaces 2007; 59: 24-34.

26. Illum L., Jabbal-Gill I., HinchcliffeM. et al. Chitosan as a novel nasal delivery system for vaccines. Adv Drug Deliv Rev. 2001; 23: 81-96.

27. Xu Y. Du Y. Effect of molecular structure of chitosan on protein delivery properties of chitosan nanoparticles. Int. J. Pharm.2003; 250: 215-226.

28. Zhu S., Qian F., Zhang Y. et al. Synthesis and characterization of PEG modified Ntrimethylaminoethylmethacrylate chitosan nanoparticles, Eur. Polym. J.2007; 43: 2244-2253.

29. Qun, G. Ajun, W. Effects of molecular weight, degree of acetylation and ionic strength on surface tension of chitosan in dilute solution Carbohydr. Polym.2006: 64: 29-36.

30.Wang W. Instability, stabilization, and formulation of liquid protein pharmaceuticals Int. J. Pharm.1999; 185: 129-188.

31. Chehin R. Thorolfsson M. Knappskog P. Domain structure and stability of human phenylalanine 
hydroxylase inferred from infrared spectroscopy.FEBS Lett. 422 .1998; 225-230.

32. McIntosh K., Charman W. Charman S. The application of capillary electrophoresis for monitoring effects of excipients on protein conformation. J. Pharm. Biomed. Anal. 16: .1998; 1097-1105.

33. Sarmento B., Martins S., Rebeiro A. et al.. Development and comparison of different nanoparticulate polyelectrolyte complexes as insulin carriers. Int. J. Peptide Res.Therap.2006; 12: 131-138.

34. Oliva A., Farina, J. Llabres, M. Influence of temperature and shaking on stability of insulin preparations: degradation kinetics. Int. J. Pharm .1996; 143: 163-170.

35. BrangeJ., Langkjaer L, Havelund, S. Hougaard, P. Chemical stability of insulin. 2. Formation of higher molecular weight transformation products during storage of pharmaceutical preparations. Pharm. Res.1992; 9: 727-734.

36. Brange J., Andersen, L., Laursen, E.D. et al. Toward understanding insulin fibrillation. J. Pharm. Sci.1997; 86: 517-525.
37. Jintapattanakit A., Junyaprasert V., Mao S. et al. Peroral delivery of insulin using chitosan derivatives: A comparative study of polyelectrolyte nanocomplexes and nanoparticles. Int. J. Pharm.2007; 342: 240-249.

38. Taluja A. Bae Y. Role of a novel excipient poly .ethylene glycol;-b-poly .L-Histidine; in retention of physical stability of insulin in aqueous solution. Pharm. Res.2007; 24: 1517-1526.

39. Wu Z., Ping Q., Song, Y. et al. Studies on the insulinliposomes double-coated by chitosan and chitosanEDTA conjugates. Yao Xue Xue Bao. 2004; 39: 933938.

40. Elsayed A, Remawi MA, Qinna N, et al. Formulation and characterization of an oily-based system for oral delivery of insulin. Eur J Pharm Biopharm. 2009; 73: 269-279.

41. Badwan A, Remawi M, Qinna N, et al. Enhancement of oral bioavailability of insulin in humans Neuroendocrinol Lett 2009; 30: 101-105. 